\title{
Mathematical approach to human reaction
}

\author{
2020/11/27 First Edition \\ Takuya Yabu(takuya.yabu@live.jp)
}

\begin{abstract}
I thought about how to get the magnitude from the event and the reaction of the other party. Evaluating the values of events and opponents' reactions using a one-dimensional random walk shows that the probability density function of the values of events and opponents' reactions has a fixed probability distribution. Similarly, I have shown that the functions that determine the magnitude of events and reactions are also represented by a fixed distribution. Therefore, I also showed that when individuals gather to form a group, the functions that determine the magnitude of events and reactions as a group are also represented by a fixed distribution. Also, as an application example of this model, I described how to show my reaction and what to do when the magnitude of the event is large.
\end{abstract}

\section{Introduction}

I wrote a paper about human emotion processing (Yabu, Mathematical approach to human emotion processing, 2020). I also wrote a paper about human character (Yabu, Mathematical approach to human character, 2020). I have shown that for an individual, there is a fixed probability distribution function that receives emotions from events, and a fixed distribution function that also has a response from the received emotions. It was also found that the group of individuals is also represented by a fixed distribution of emotions and reactions as a group. For individuals, the probability density function representing character has been shown to have a fixed probability distribution. It was also found that even in a group of individuals, the distribution that expresses the character of the group is also represented by a fixed distribution. Although it has become possible to mathematically explain emotion processing and character, the part that determines its magnitude remains unclear from actual events or reactions of the other party.

So, I mathematically thought about how the magnitude of actual events or reactions of the other party are determined and how I should deal with my reaction because my reaction is exaggerated.

2. About an individual's reaction to an event or the reaction of another person

In the paper I wrote (Yabu, Mathematical approach to human emotion processing, 
2020), I described human emotion processing using a mathematical model that receives emotions $x$ from events $X$ and responds $F(x)$ to those emotions $x$ received. Figure 1 shows the relationship.

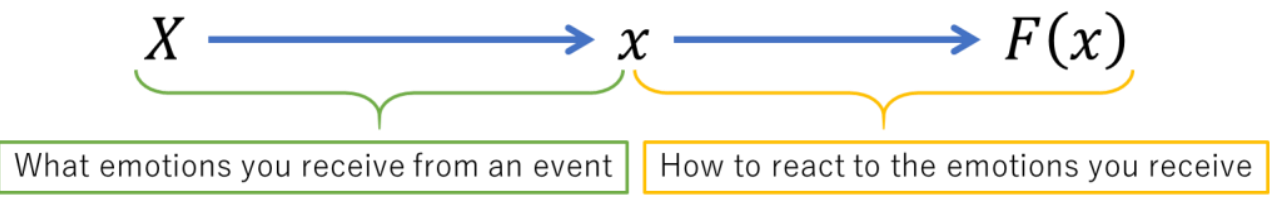

Figure 1 About the relationship between events, emotions, and reactions

In my mathematical model, the emotion $\mathrm{x}$ was determined from the following relationship between the event and the reaction function.

$$
X=F(x)
$$

This emotional choice determines the emotions one receive, which in turn determines the magnitude of one's next reaction. I mentioned that the function that determines the magnitude of the reaction from the received emotion is determined by the following function in the case of an individual.

$$
F(x)=\frac{1}{\sqrt{2 \pi}} e^{-\frac{x^{2}}{2}}
$$

Here, equation ( 2) expresses the magnitude of the reaction. I have not modeled how one reacts.

In equation ( 1 ), we defined and mathematically stated that personality is responsible for selecting emotions with positive or negative signs (Yabu, Mathematical approach to human character, 2020). I also defined that how an individual reacts is automatically determined by what kind of event is occurred and what kind of emotion is received.

Consider a variable called $r$ for an event or an individual's reaction, and add 1 if there is a favorable point and -1 if there is an unfavorable point from $r=0$. In other words, I will perform a one-dimensional random walk for events or reactions shown by individuals. In other words, the discussion of emotion processing (Yabu, Mathematical approach to human emotion processing, 2020) can be used as it is. The probability density function $p_{\text {event }}(r)$, which selects the value $r$ of an event or reaction by an individual, is expressed by the following formula as in emotion processing.

$$
p_{\text {event }}(r)=\frac{1}{\sqrt{2 \pi}} e^{-\frac{r^{2}}{2}}
$$

That is, the choice of $r$ for an event or an individual's reaction is represented by a standard normal distribution.

For this equation ( 3 ), considering that the larger the probability, the larger the 
magnitude of the reaction, the following equation can be obtained. It is the same as defining the reaction function using the proportionality constant $q_{0}$ used when defining the reaction function in the paper about emotion processing (Yabu, Mathematical approach to human emotion processing, 2020).

$$
X(r)=\frac{q_{0}}{\sqrt{2 \pi}} e^{-\frac{r^{2}}{2}}
$$

This formula determines the magnitude of the reaction to $\mathrm{r}$ for the event or the reaction indicated by the individual. Regarding this $q_{0}$, we can have the same discussion as the emotion processing paper, and $q_{0} \rightarrow 1$. Therefore, when an individual receives $r$ from an event or a reaction indicated by the individual, the magnitude is determined by the following function. I define this as an event function.

$$
X(r)=\frac{1}{\sqrt{2 \pi}} e^{-\frac{r^{2}}{2}}
$$

In other words, the magnitude of the reaction $r$ indicated by an event or individual is represented by a standard normal distribution.

3. About the reaction of the group to the events and the reactions of others Now, it turns out that the individual event function is expressed by equation ( 5 ).

Therefore, we follow the magnitude of reaction of group is the following formula, which is the same event function as an individual. It is the same as discussing response of a group of individuals to the events or the reaction of others in the above paper (Yabu, Mathematical approach to human emotion processing, 2020). This is the group event function.

$$
X_{\text {group }}(r)=\frac{1}{\sqrt{2 \pi}} e^{-\frac{r^{2}}{2}}
$$

In other words, the event functions of individuals and groups are the same. The graph of equation (6) is as follows. 


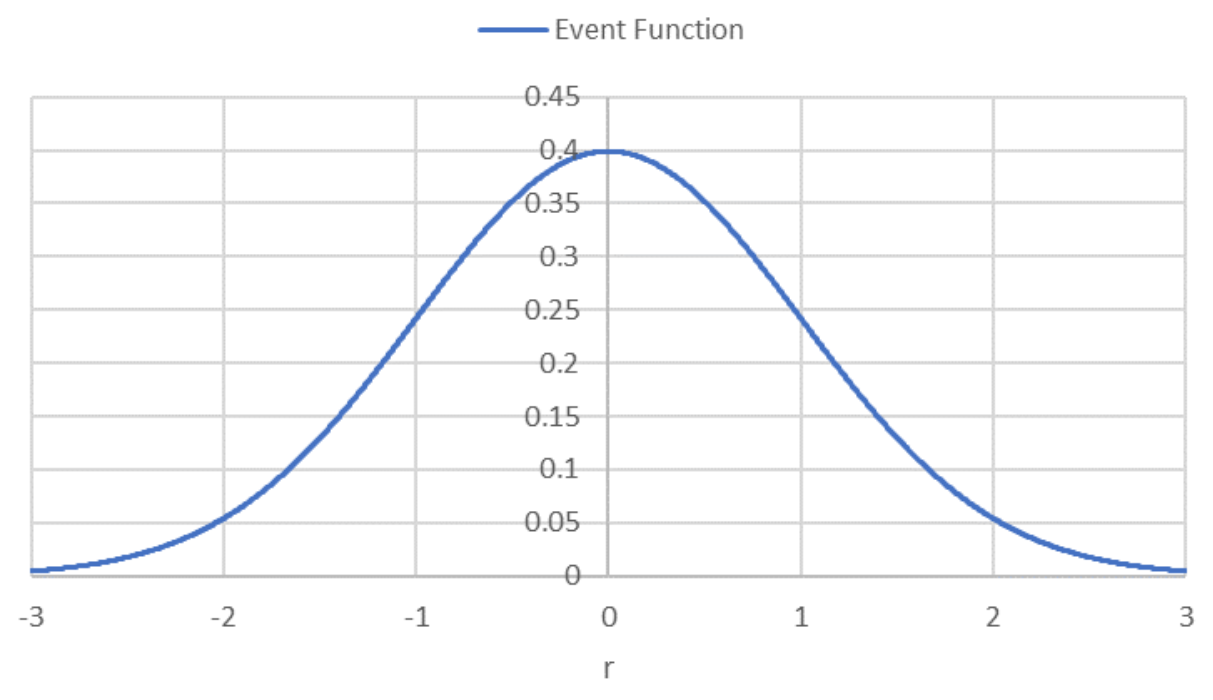

Figure 2 Individual and group event function

Since the event function of an individual and a group is the same, the same reaction magnitude value is given for the same event or individual / group reaction. Therefore, we can see that individuals and groups have the same standards and can be controlled as a group. I think that the mathematical model of the event function presented can explain the reality.

4. How to deal with one's reaction

In my mathematical model of event function, every human has the same event function. In addition, when considered together with the emotion processing paper (Yabu, Mathematical approach to human emotion processing, 2020), if it shows an exaggerated reaction, it is easy to judge that $r$ is big, the event function becomes a small value, and from equation ( 1 ), the other party will generate small positive or negative emotions. On the contrary, if it shows a calm reaction, it is easy to judge that $r$ is small, the event function becomes a large value, and from equation (1), the other party will generate small positive or negative emotions. In the character paper (Yabu, Mathematical approach to human character, 2020), $x=0$ means a situation of convincing, reassuring, stable, and emotionless.

From these facts, it can be seen that when there is any reaction, it is better to calm down and take a small action before reacting. As a result, the other party feels at ease. We also find that the following properties of the sum of events (Yabu, Mathematical approach to human emotion processing, 2020) suggest that it is best not to repeat the same behavior many times in a short period of time. Note that the value of $X_{0}$ in 
equation ( 7) is determined by equations ( 5) and( 6). Here, $\tau$ is the value obtained by subtracting the period judged to be important from the period judged to be insignificant (the unit is seconds, but 0 if it is 0 or less).

$$
X=X_{0} e^{-\frac{\tau_{1}^{2}}{2}}+X_{0} e^{-\frac{\tau_{2}^{2}}{2}}+\cdots+X_{0} e^{-\frac{\tau_{n}^{2}}{2}}=n X_{0} e^{-\frac{\tau_{1}^{2}+\cdots+\tau_{n}^{2}}{2 n}}
$$

In other words, it is mathematically not good to think that you have done something very sorry to the other person and try to continue to apologize in a loud voice over and over again, "I'm sorry. I beg your pardon" like me. Again, it's a good idea to calm down and take smaller actions before reacting anyway.

That's how I can deal with my reaction. I also practice. I will report any changes.

\section{About the reaction when a big event occurs}

Think about a big event, such as a depression caused by multiple actions or behaviors that cannot be handled, a big accident or illness, or a big natural disaster.

In this case, think of a big event as a collection of small events. In the case of depression, each word and action corresponds to it. Even a big accident or illness is a combination of various events such as physical pain, surgery, and the need for money. So is a big natural disaster.

These small events are sized by equations (5) and (6), but by adding them as in equation ( 7), As I wrote in emotion processing paper (Yabu, Mathematical approach to human emotion processing, 2020), it is mathematically thought that the magnitude of the event will exceed $\frac{1}{\sqrt{2 \pi}}$, and it will be rejected, ignored, or became depression.

I also wrote about these in the emotion processing text. If you are depressed, escape from the situation. In the case of a big accident, illness or big natural disaster, spend more time not thinking about it. By doing so, I think it would be better to make the magnitude of the event smaller than equation ( 7).

\section{Conclusion}

I considered how humans respond to events and individual's reactions. I considered a variable called $r$ for the event or the reaction of individual, and modeled the part that evaluates the event or the reaction of individual by a one-dimensional random walk. As a result, it was found that the probability density function of the reaction $r$ indicated by an event or an individual is represented by a standard normal distribution. I also found that by using the probability density function and the proportionality constant to define an event function that represents the magnitude of an event or reaction indicated 
by an individual, the event function is also represented by a standard normal distribution. It also showed that the event function of a group of individuals is also represented by a standard normal distribution. This shows that the group can take control because the event function of the individual and the group is the same, so that the evaluation of the magnitude of the event or reaction can be unified. From the above, it was suggested that when the other person's emotions are reassuring, which is close to $x=0$, it is better to calmly react small and not repeat. Finally, I talked about what to do if a big event happens. I will try the proposed method myself. I will report any progress. It would be an unexpected joy if it helps.

\section{References}

Yabu, T. (2020). Mathematical approach to human character. Retrieved from psyarxiv.com/yrd9v

Yabu, T. (2020). Mathematical approach to human emotion processing. Retrieved from psyarxiv.com $/ \mathrm{k} 3 \mathrm{j} 4 \mathrm{z}$ 